\title{
Accuracy of the Hartree-Fock method for Wigner molecules at high magnetic fields
}

\author{
B. Szafran,${ }^{1,2}$ S. Bednarek,${ }^{2}$ J. Adamowski, ${ }^{2}$ M.B. Tavernier, ${ }^{1}$ Egidijus Anisimovas,${ }^{1}$ and F.M. Peeters ${ }^{1}$ \\ ${ }^{1}$ Departement Natuurkunde, Universiteit Antwerpen (UIA), B-2610 Antwerpen, Belgium \\ ${ }^{2}$ Faculty of Physics and Nuclear Techniques, AGH University of Science and Technology, Kraków, Poland
}

\begin{abstract}
Few-electron systems confined in two-dimensional parabolic quantum dots at high magnetic fields are studied by the Hartree-Fock (HF) and exact diagonalization methods. A generalized multicenter Gaussian basis is proposed in the HF method. A comparison of the HF and exact results allows us to discuss the relevance of the symmetry of the charge density distribution for the accuracy of the HF method. It is shown that the energy estimates obtained with the broken-symmetry HF wave functions become exact in the infinite magnetic-field limit. In this limit the charge density of the broken-symmetry solution can be identified with the classical charge distribution.
\end{abstract}

\section{INTRODUCTION}

Properties of electron systems confined in quantum dots at high magnetic fields have recently become a subject of intensive theoretical studies $1,2,3,4,5,6,7,8,9,10,11,12,13,14,15,16,17,18,19,20,21,22$.

These studies were inspired on the one hand by the experimental investigation of the addition spectra of vertical quantum $\operatorname{dots}^{23}$ at high magnetic fields, which revealed a rich structure of magnetic-field induced ground-state transformations in the confined electron system $1,2,3,4,5,6,7,8,9,10,11,12,13,14,15,16,17,18,19,20,21,22,23,24,25,26$,

and on the other hand by the search for a new symmetry in few-electron systems. One of the most interesting problems in this research is the possibility of the formation of Wigner molecules $2,3,4,5,6,7,8,9,10,25,26,27$ in which the confined electrons are distinctly spatially separated. Previous theoretical studies are based on the Hartree-Fock (HF) method 2.3.4.5.6.8, density functional theory (DFT) ${ }^{9}$, and the exact diagonalization (ED) scheme $11,12,13,14,15,16,17,18,19,20,21$. The model confinement potential used most commonly 1.2.3.4.5.6.7.8.9.10.11.12.13.14.15.16.17.18.19 is the two-dimensional (2D) cylindrically symmetric harmonic oscillator potential, which is a reasonable approximation of the confinement potential28 in vertical quantum $\operatorname{dots}^{23}$.

The external magnetic field induces ground-state transformations in the quantum-dot confined $N$-electron system, which are associated with changes of the total angular momentum and the total spin. At a certain magnetic field the electrons become spin polarized and occupy the lowest-energy Fock-Darwin states with the $z$ component of the angular momentum changing from 0 to $(1-N) \hbar^{10}$. This state is called a maximum density droplet (MDD) $\frac{1}{\underline{1}}$. In the MDD the $z$ components of the total spin and total angular momentum take on the absolute values $N \hbar / 2$ and $N(N-1) \hbar / 2$, respectively. The MDD ground state is predicted by the ED method $16,17,18,19,20$ as well as by the $\mathrm{HF}^{2}$ and $\mathrm{DFT}^{9}$ methods. Higher magnetic fields lead to a decay of the MDD, which has been observed experimentally ${ }^{23}$. This decay, considered in the framework of the ED method ${ }^{16,17}$, is related to the increase of the absolute value of the total angular momentum above the value corresponding to the MDD phase. The unrestricted $\mathrm{HF}^{2,3,4,5}$ and $\mathrm{DFT}^{9}$ methods predict that the ground state of the electron phase created after the decay of the MDD possesses a charge density which does not reproduce the symmetry of the external confinement potential. These states will be called "broken-symmetry states" throughout the present paper. In the brokensymmetry solutions obtained by the HF and DFT methods, the electrons become localized at separate space sites forming a Wigner molecule 2 .5.6.7.9.10.25.26.27. In the ED method the separation of the electrons, i.e., the formation of the Wigner molecules, appears in the relative coordinates of the electron system and is not necessarily related to the broken symmetry of the charge density ${ }^{10}$. The HF broken-symmetry solutions are not eigenfunctions of the angular momentum operator, but are degenerate with respect to rotations, i.e., can be oriented at an arbitrary angle. The rotational symmetry of the HF broken-symmetry solutions for the few-electron system can be restored with a post-HF treatment ${ }^{4}$.

A multicenter basis with the one-electron wave functions was used in the theoretical studies of the 2D Wigner crystals 29.30 . Recently, a similar multicenter basis was used 4.5.6.7 to study the quantum-dot confined electron systems. The papers ${ }^{4.5 .6 .7}$ were based on the unrestricted HF method ${ }^{4,5,6}$ and the variational method 7 with the trial wave function in the form of a single Slater determinant with non-orthogonal one-electron wave functions. These one-electron wave functions ${ }^{4.5 .6 .7}$ at high magnetic field yield point-like charge density distributions. Therefore, in the limit of infinite magnetic field, the groundstate charge density, obtained with the multicenter basis, is identical with the lowest-energy classical configuration of the point charges $31,32.33$. The multicenter basis is a very efficient tool for the investigation of the Wigner molecules, since it requires only a single basis function per electron, while the convergence of the HF energy estimates in the one-center Fock-Darwin basis ${ }^{2}$ is very slow in the regime of the island-like Wigner localization. Using the unrestricted $\mathrm{HF}$ method with the multicenter basis (MCHF), Szafran et $a l^{5}$ have shown that the external magnetic field leads to transformations of the groundstate symmetry of the Wigner molecules. Only in the 
high magnetic field limit the ground-state phase (isomer of the Wigner molecule) $)^{\frac{5}{5}}$ corresponds to the configuration of electrons, which is identical with that of a classical system of point charges 31.32 .33 .

In the present paper, we perform a detailed study of the physics behind the formation of the Wigner molecules. In particular, we discuss the accuracy of the broken symmetry solutions obtained with the multicenter basis in comparison with the ED results for two, three, and four electrons. The physical interest of this study relies on the investigation of the quantum systems in a classical localization limit.

The paper is organized as follows: in Section II we briefly describe the theoretical methods, in Section III we present our numerical results, in Section IV - the discussion, and in Section $\mathrm{V}$ - the conclusions and the summary. In Appendix, we derive the wave function used in the present calculations.

\section{THEORY}

We consider the $N$-electron system confined in the 2D harmonic oscillator potential with frequency $\omega_{0}$, subject to the external magnetic field $B$ oriented perpendicularly to the quantum dot plane ${ }^{5.6}$. We apply the MCHF and ED methods. In the MCHF method we assume that all the electrons are spin polarized by the magnetic field and apply the Landau gauge, i.e., $\mathbf{A}=(-B y, 0,0)$. We expand one-electron wave function $\Psi_{\mu}(\mathbf{r})$ of the $\mu$-th occupied state $(\mu=1, \ldots, N)$

$$
\Psi_{\mu}(\mathbf{r})=\sum_{i=1}^{N} c_{i}^{\mu} \psi_{\mathbf{R}_{i}}(\mathbf{r}),
$$

in the basis

$$
\begin{gathered}
\psi_{\mathbf{R}}(\mathbf{r})=(\alpha / 2 \pi)^{1 / 2} \exp \left\{-(\alpha / 4)(\mathbf{r}-\mathbf{R})^{2}+\right. \\
(i \beta / 2)(x-X)(y+Y)\}
\end{gathered}
$$

where $\mathbf{R}=(X, Y), \mathbf{r}=(x, y)$, and $\alpha$ and $\beta$ are treated as nonlinear variational parameters. Function (2) with $\alpha=\beta=m^{*} \omega_{c} / \hbar$ is the wave function of the lowest Landau level $\left(\omega_{c}=e B / m^{*}\right.$ is the cyclotron frequency and $m^{*}$ is the electron effective mass). Moreover, function (2) with $\alpha=\left(2 m^{*} / \hbar\right) \sqrt{\omega_{0}^{2}+\omega_{c}^{2} / 4}$ and $\beta=m^{*} \omega_{c} / \hbar=e B / \hbar$ is the eigenfunction of the Fock-Darwin ground state for the lateral parabolic confinement potential centered at point $\mathbf{R}$ with the energy equal to $\hbar \sqrt{\omega_{0}^{2}+\omega_{c}^{2} / 4}$ (see Appendix). The probability density associated with wave function (2) is the Gaussian centered around point $\mathbf{R}$. The centers of basis functions (2) are taken from scaled configurations $\left\{\mathbf{R}^{\text {class }}\right\}$ of classical Wigner molecules, i.e., $\{\mathbf{R}\} \equiv \mathbf{R}_{i}=\sigma \mathbf{R}_{i}^{\text {class }}$, where $i=1, \ldots, N^{5.6}$. The scaling parameter $\sigma$ is the third nonlinear variational parameter used in the present approach. In Refs. [5,6] only two nonlinear variational parameters were used, namely, the scaling parameter $\sigma$ and a single variational parameter $\alpha=\beta$. In the following, we will show that the introduction of the two independent variational parameters in the real and imaginary part of the exponent in Eq.(2) leads to a significant improvement of the variational energy estimates at finite magnetic field. Throughout the present paper, the basis with the restriction $\alpha=\beta$ will be referred to as "restricted" and without it as "generalized".

In the present paper we also apply the ED method. The ED results, that in principle are the exact solutions of the few-electron Schrödinger equation, are used as reference data for the estimation of the accuracy of the MCHF results. In the ED calculations we use the symmetric gauge $[\mathbf{A}=(-B y / 2, B x / 2,0)]$, that allows us to exploit the angular symmetry of the one-electron wave functions. The Schrödinger equation for the $N$-electron system can be separated into the center-of-mass and relative-coordinate equations 15.19 .22 . The center-of-mass eigenproblem possesses an analytical solution. For $N=2$ the relative-motion eigenproblem can be easily solved numerically with an arbitrary precision using a onedimensional finite difference method. This approach ${ }^{15.19}$, used in the present paper for the two-electron system, is not applicable to the systems with a larger number of electrons. Thus, for $N=3$ and $N=4$ the ED procedure is constructed according to the configuration interaction method. First, we solve the Schrödinger equation for a single noninteracting electron with a definite angular momentum using a finite-difference approach on the one-dimensional mesh with 200 points. Next, we use the single-electron wave functions to construct Slater determinants with the required total angular momentum and spin. The $N$-electron Schrödinger equation is diagonalized in the orthonormal basis of Slater determinants with proper spin-orbital symmetry and the Coulomb matrix elements are integrated numerically. The basis, i.e., the choice of the single-electron wave functions forming the Slater determinants, is optimized separately for each state. The calculations have been performed with a precision better than $0.01 \mathrm{meV}$. For $N=4$ this precision requires the application of the basis containing up to 2000 Slater determinants. The ED calculations of the ground state for $N=3$ and 4 are carried out up to $20 \mathrm{~T}$ with the maximal absolute values of the angular momentum equal to 18 and $34 \hbar$, respectively.

\section{RESULTS}

Let us first discuss the corrections to the MCHF energy estimates obtained with the generalized wave function (2). We use as an example the system of six confined electrons. Figure 1 shows the difference between the energy estimates obtained with and without the restriction $\alpha=\beta$ in wave function (2). In the calculations we use the material parameters of GaAs, i.e. $m^{*}=0.067 m_{e}$, dielectric constant $\varepsilon=12.9$, the effective Landè factor 


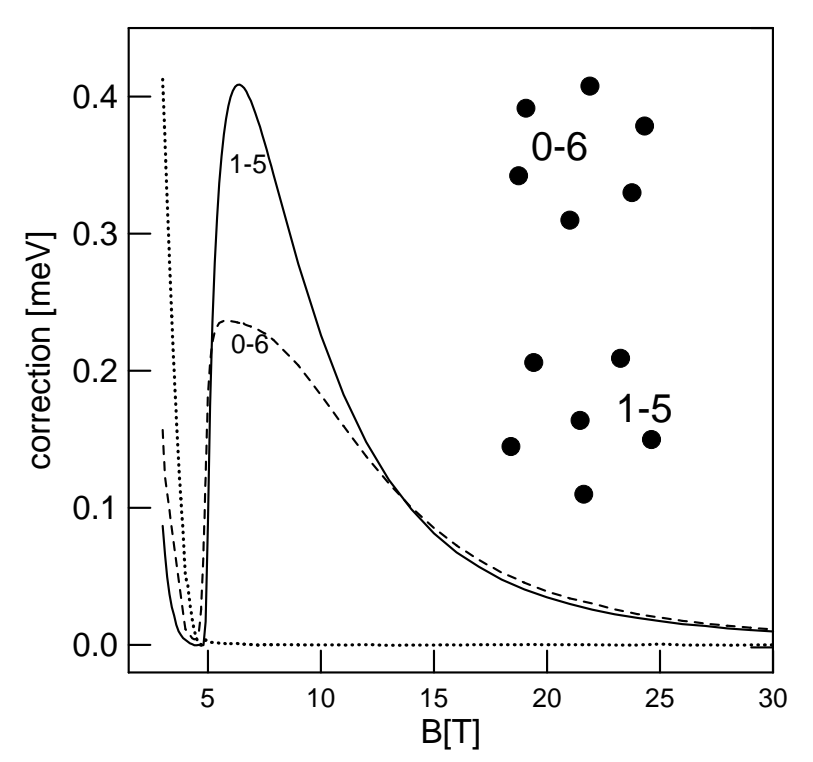

FIG. 1: Correction to the energy estimates obtained with the generalized MCHF method and calculated with respect to the results of the restricted HF method $(\alpha=\beta)$ for the sixelectron system with the configuration 1-5 (solid line) and 0-6 (dashed line) as a function of magnetic field $B$. The dotted line shows the overestimation of the energy obtained for 1-5 configuration with the value of $\beta$ fixed at $e B / \hbar$. Inset: the classical 0-6 and 1-5 Wigner molecules.

$g^{*}=-0.44$, and assume the confinement energy $\hbar \omega_{0}=3$ $\mathrm{meV}^{2.5}$. The centers of basis (1) are taken from scaled classical configurations 1-5 and 0-6 [the phases (isomers) of the Wigner molecule are labelled by the numbers of electrons localized in the subsequent rings starting from the innermost one]. Phase $0-6$ is the one, in which the Wigner molecule is created ${ }^{5,10,14}$ after the MDD breakdown, and possesses an intermediate character. The 15 configuration is the lowest-energy configuration of the classical Wigner molecule ${ }^{32}$. This is also the groundstate configuration of the six-electron quantum system at high magnetic field ${ }^{5}$. In the magnetic field below 4.9 $\mathrm{T}$ the mutlicenter bases with both the 0-6 and 1-5 configurations mimic the cylindrically symmetric MDD charge distribution 5 . The correction obtained with the generalized basis possesses a minimum in the magnetic field inducing the MDD breakdown, for which, the application of the generalized basis does not improve the results. However, the generalized basis leads to considerable corrections to the energy both in the MDD stability regime and after the MDD breakdown. This correction falls down to zero in the high-magnetic field, in which both the generalized and restricted bases work with nearly the same precision.

The dotted line in Fig. 1] shows the overestimation of the energy obtained for 1-5 configuration with fixed value of parameter $\beta=e B / \hbar$. The value of $\beta$ has an influence

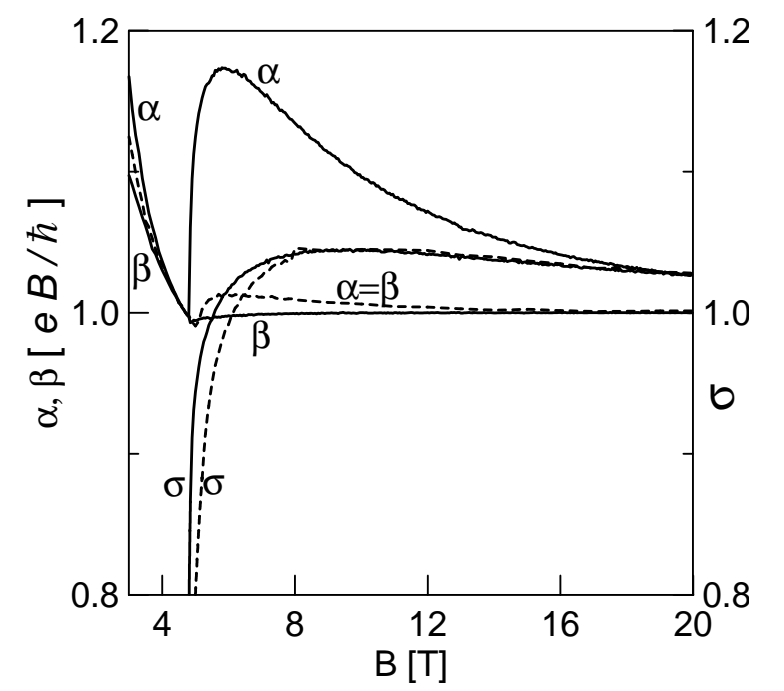

FIG. 2: Optimal values of the variational parameters for the 1-5 configuration obtained with the generalized ( $\alpha \neq \beta$, solid lines) and restricted ( $\alpha=\beta$, dashed lines) basis. Parameters $\alpha$ and $\beta$ are expressed in units $e B / \hbar$, parameter $\sigma$ is dimensionless.

on the MCHF charge density due to the interference of the single-electron wave functions [Eqs. (12)] centered at different sites. Fig. 1 shows that the variation of $\beta$ has a large influence on the estimates of the energy in the MDD phase. On the other hand, in the Wigner molecule phase, the value of $\beta$ can be safely put equal to $e B / \hbar$.

Figure 2 shows the magnetic-field dependence of the optimal variational parameters obtained for the 1-5 configuration in expansion (11). The dependence of the scaling parameter $\sigma$ is qualitatively the same for both wave functions. This parameter grows rapidly when the MDD breaks down into the molecular phase. Generalized wave function (2) with $\alpha \neq \beta$ leads to the MDD decay at lower values of the magnetic field, which is visible in the dependence of $\sigma$ on the magnetic field. Parameter $\sigma$ tends to 1 at high magnetic fields for which the quantum Wigner molecule takes the shape and size of its classical analog, i.e., $\{\mathbf{R}\} \longrightarrow\left\{\mathbf{R}^{\text {class }}\right\}$. Parameters $\alpha$ and $\beta$ decrease with the increasing magnetic field in the MDD regime (cf. solid lines for $B<\sim 5 \mathrm{~T}$ in Figure 2). Just before the MDD breakdown $\alpha$ and $\beta$ take on very close values, which leads to the minimal overestimation of the energy obtained with restriction $\alpha=\beta$ at the MDD breakdown (cf. Fig. 1). We have found that the increase of $\beta$ above $e B / \hbar$ in the MDD regime makes the local maxima of the charge density 'sink' in the global flat maximum characteristic ${ }^{6}$ for the MDD phase. After the MDD breakdown parameter $\beta$ quickly reaches $e B / \hbar$, i.e. takes on the value which corresponds to both the lowest Landau and Fock-Darwin levels [cf. the discussion of wave function (2) in Sec. II]. At high magnetic fields the wave functions (2) centered around different sites stop to 
overlap; in consequence, $\beta$ stops to influence the $\mathrm{MCHF}$ charge density and takes the value $e B / \hbar$, which ensures the equivalence of the centers of Landau orbitals. The present finding that the parameter $\beta$ becomes equal to $e B / \hbar$ just after the MDD breakdown, in spite of the nonvanishing overlap is not evident a priori. We can give the following physical interpretation to this finding: the electrons in the Wigner molecule behave as if they occupied the independent one-particle Fock-Darwin ground-state orbitals, each of them centered around its own local minimum of the potential energy. The optimal value of $\alpha$ rapidly grows after the MDD breakdown (cf. Fig. 2), which leads to the lowering of the energy obtained in the Wigner molecule regime $(B>5 \mathrm{~T})$. The increase of $\alpha$ above $e B / \hbar$ enhances the electron localization and lowers the electron-electron interaction energy.

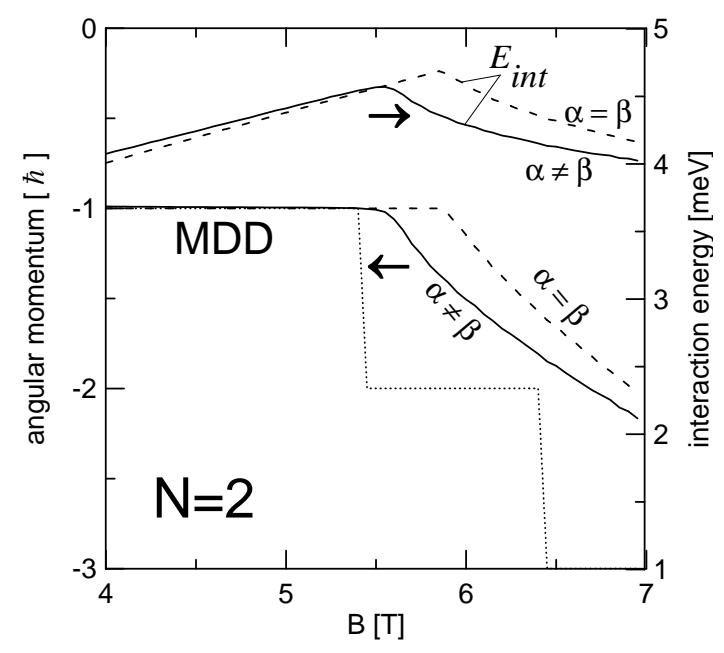

FIG. 3: Total angular momentum (left scale) of the exact ground state of the two-electron system (dotted line) and MCHF expectation values calculated with the generalized ( $\alpha \neq \beta$, solid line) and the restricted $(\alpha=\beta$, dashed line) basis. The two curves marked by $E_{\text {int }}$ show the expectation value of the electron-electron interaction energy (right scale) calculated with the MCHF methods.

Figure 3 illustrates the MDD decay picture obtained with the ED and MCHF methods for the two-electron system. The dotted line shows the exact values of the $z$ component of the total angular momentum as a function of the magnetic field. The decay of the MDD is related with a stepwise decrease of the angular momentum from $-\hbar$ to $-2 \hbar$, which appears for $B=5.45 \mathrm{~T}$. The solid (dashed) line shows the expectation value of the total angular momentum obtained with the MCHF method using the generalized (restricted) basis. Figure 3 shows that within the MDD stability regime both the present MCHF methods reproduce the exact value of the angular momentum. When the MDD breaks down, the MCHF expectation value of the angular momentum decreases monotonically with the increasing $B$, in contrast to the exact stepwise behavior. The MDD breakdown ob-

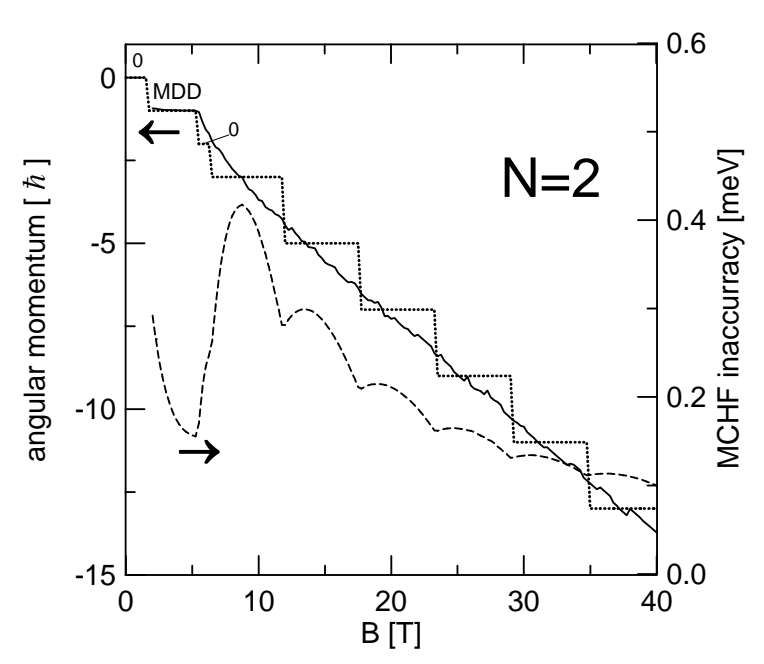

FIG. 4: Total angular momentum (left scale) of the exact ground state of the two-electron system (dotted line) and the MCHF expectation value calculated with the generalized basis (solid line) as functions of magnetic field $B$. The states with unpolarized spins are marked by "0". The dashed line shows the difference of the ground-state energy obtained with the $\mathrm{MCHF}$ and the exact energy calculated with the ED scheme (right scale).

tained by the HF method is also related to a cusp of the interaction energy ${ }^{\underline{6}}$ (cf. two upper curves in Fig. 3). In the MDD regime the charge density distribution shrinks with increasing magnetic field, which results in an increase of the interaction energy. The transformation of the charge density from the droplet into the molecular phase occurs when the interaction energy exceeds some threshold value. The MCHF method with the generalized (restricted) basis leads to a MDD breakdown for $\mathrm{B}=5.55$ $\mathrm{T}(5.85 \mathrm{~T})$.

Figure 4 displays the exact ground-state angular momentum for the two-electron system (dotted line) and the expectation value obtained within the HF method with the generalized multicenter basis (solid line) as well as the difference between the MCHF and the exact energy (dashed line). The $z$ component of the total spin is equal to $\hbar$ with the exception of the low-magnetic field ground state and the state which appears just after the MDD breakdown. These two states possess zero spin and are labelled by " 0 " in Figure 4 Notice that the expectation value of the total angular momentum follows quite well the exact value. Moreover, the overestimation of the ground state energy within the MCHF method decreases with increasing magnetic field. This decrease is non-monotonous, and the MCHF inaccuracy exhibit local minima for magnetic fields for which the exact angular momentum changes.

The origin of this oscillatory behavior is explained in Fig. 5] which shows the MCHF energy estimate and the exact ground-state energy of the two-electron system 


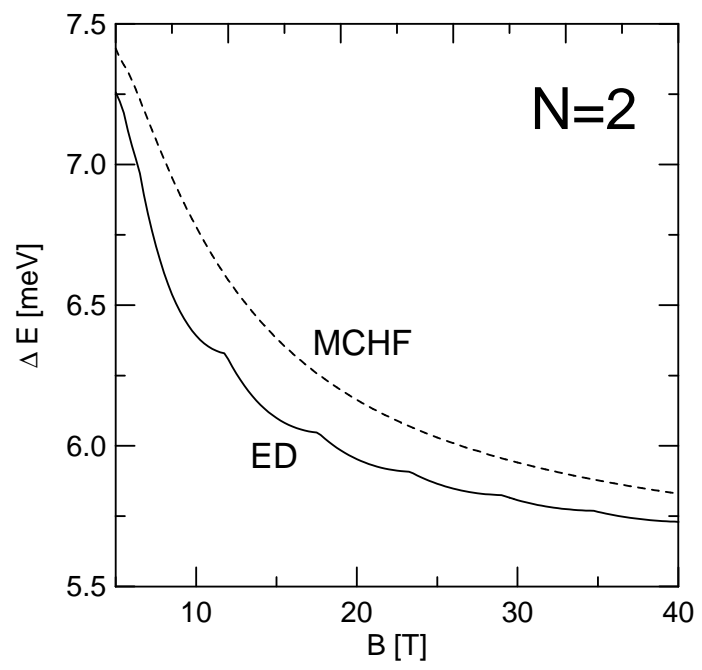

FIG. 5: Energy $\Delta E$ of the two-electron system calculated with respect to the lowest Landau level as a function of magnetic field $B$. Solid (dashed) curve show the exact (MCHF) results.

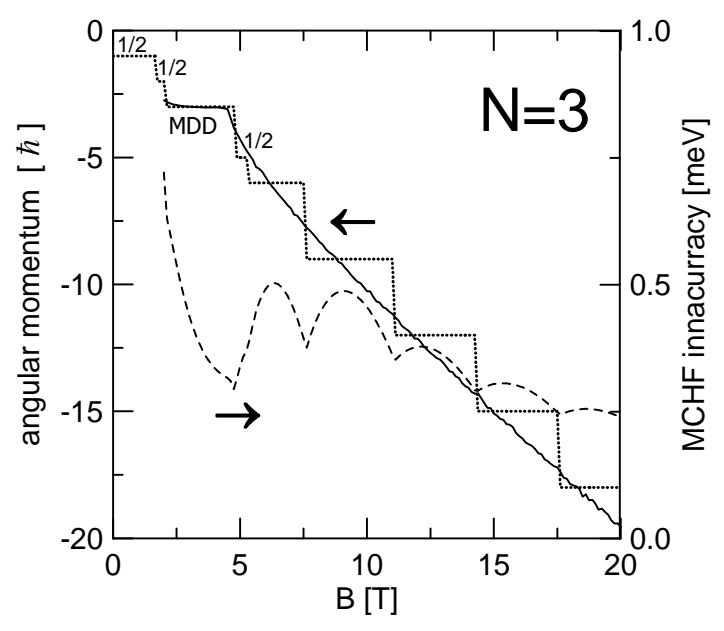

FIG. 6: Same as Figure 4 but now for the three-electron system. The states with unpolarized spins are marked with "1/2".

calculated with respect to the lowest Landau level, i.e., $\Delta E=E-2 \times\left(\hbar \omega_{c} / 2+\hbar g^{*} \mu_{B} B / 2\right)$. The MCHF estimate is a smooth function of the magnetic field, while the exact energy possesses cusps at the magnetic fields at which the ground-state angular momentum changes abruptly. For these magnetic fields the MCHF estimate is visibly closer to the exact energy value, which explains the local minima in Figure 4.

A similar comparative study between the exact and the MCHF results has been made for the $N=3$ and $N=4$ systems. For the system of three electrons the results are shown in Fig. 6, in which the ground states with the total spin equal to $\hbar / 2$ are labelled by " $1 / 2$ ". The other states are fully spin polarized. Similarly as in the case of the two-electron system the ground state which appears after the MDD breakdown is not spin polarized. The overestimation of the total energy obtained with the MCHF method exhibits a similar qualitative dependence on the magnetic field as for two electrons. In contrast to the two-electron case, the MCHF method with the generalized basis predicts a breakdown of the MDD for a slightly smaller magnetic field value $(B=4.6 \mathrm{~T})$ than the exact result $(B=4.8 \mathrm{~T})$. The MCHF with the restricted basis yields the magnetic field inducing the MDD breakdown $B=5 \mathrm{~T}$.

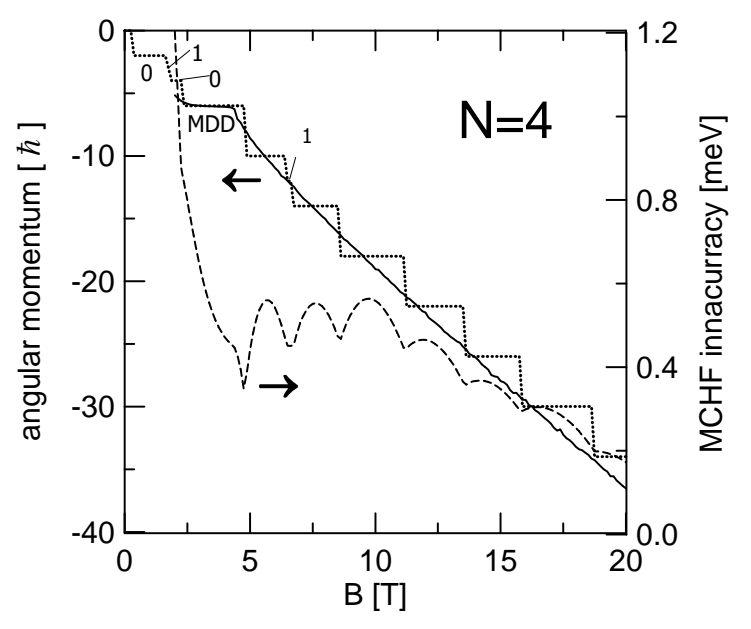

FIG. 7: Same as Figure 4 but now for the four-electron system. The states with unpolarized spins are marked with the value of the $z$-component of the total spin in $\hbar$ units.

A similar result for the four-electron quantum dot is shown in Figure 7 The non-fully-polarized ground states are marked by the quantum numbers of the total-spin $z$ component "0" and "1". Contrary to the two and three electron systems, the four-electron MDD decays into a spin-polarized state, but a low-spin state still appears at the higher magnetic field. The transitional appearance of low-spin ground states at magnetic fields after the MDD breakdown have been reported first in Ref ${ }^{11}$ for $N=2$ and $N=4$. The exact magnetic field inducing the MDD decay is equal to $4.75 \mathrm{~T}$, while the MCHF with the generalized basis predicts a value of $4.38 \mathrm{~T}$ and the MCHF with the restricted basis gives $4.8 \mathrm{~T}$. In this case the MCHF with the restricted basis gives accidentally a better estimate for the MDD breakdown field. For five electrons the difference between both the MCHF estimates of the magnetic field inducing the MDD decay is $0.4 \mathrm{~T}$. For six and more electrons the differences are not larger than 0.2 T.

Figures 4, 6, and 7 show that the MCHF inaccuracy decreases with the increasing magnetic field. In order to find the high-field asymptotic behavior of the MCHF energy estimate we have plotted in Figure 8 the MCHF 


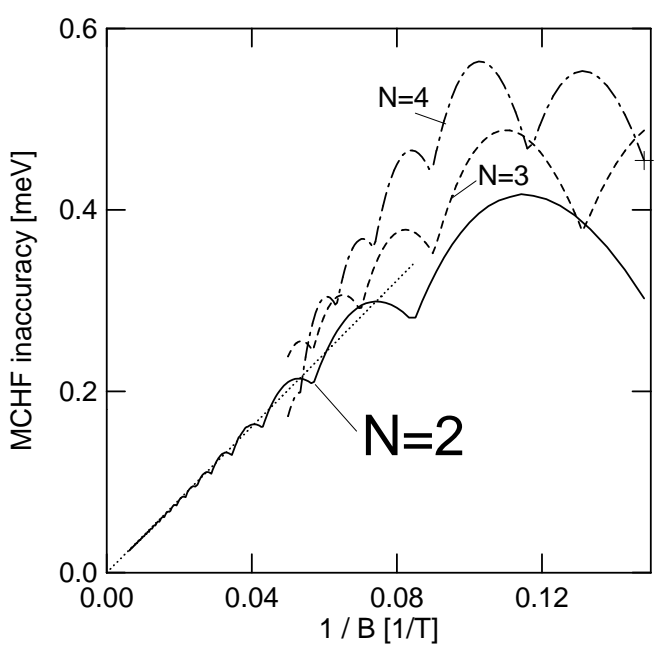

FIG. 8: The MCHF inaccuracy for the ground state for the two-electron system as a function of $1 / B$ for $N=2$ (solid line), 3 (dashed line) and 4 (dash-dotted line) The dotted line shows the high-magnetic field asymptote for $N=2$, parametrized as $4.026 / B[\mathrm{meV} / \mathrm{T}]$.

error as a function of $1 / B$ for $N=2,3$, and 4 . The plot for two electrons covers the magnetic fields up to $160 \mathrm{~T}$, while the plots for three and four electrons are drawn up to $20 \mathrm{~T}$ only. The results for $N=2$ show that at high magnetic fields the MCHF inaccuracy is proportional to $1 / B$. At high magnetic fields the asymptotic behavior of the MCHF inaccuracy for $N=2$ can be very well approximated by the function $f(B)=4.026 / B[\mathrm{meV} / \mathrm{T}]$, and consequently the MCHF approach becomes exact for $B \rightarrow \infty$. The plots for $N=3$ and 4 in the studied (narrower) range of the magnetic field exhibit a similar tendency as that for $N=2$, however, they do not become linear functions of $1 / B$ for $B<20 \mathrm{~T}$. The comparison of the MCHF inaccuracies for $N=2,3$, and 4 indicates that the overestimation of the MCHF ground-state energy in the magnetic field range above the MDD instability does not substantially increase with $N$. For $B=20 \mathrm{~T}$ the overestimation of the ground-state energy for $N=2,3$, and 4 is equal to $0.21,0.24$, and $0.18 \mathrm{meV}$, respectively.

\section{DISCUSSION}

Figs. 4] 6] and 7 show that at high magnetic field the exact ground-state angular momentum take the so-called magic values $25,34.35 .36$ and change by $N \hbar$. Only for these magic values of the angular momenta the classical symmetry can be reproduced in the inner coordinates of the quantum systems ${ }^{25}$. On the other hand the classical symmetry is ensured in the MCHF by the present choice of centers of orbitals (2) and the linear change of the expectation value of the total angular momentum is related with the growing localization of wave functions (2).
The results presented in Figs. 4, 6] 17 and 8 show that the broken-symmetry solutions obtained with the MCHF method provide exact energy results in the high magnetic field limit. This fact might be rather surprising, since one could expect that the exact solutions of the few-electron Schrödinger equation should also be the eigenfunctions of the angular momentum operator. The exact eigenfunctions yield the charge density distribution, which reproduces the symmetry of the confinement potential. This apparent contradiction can be solved if we consider the Schrödinger equation for the electron system confined in the parabolic potential. This equation can be separated in the center-of-mass and relative coordinates. In the framework of the ED approach, the separation of the electrons, i.e., the Wigner localization, appears in the relative (inner) coordinates of the electron system, while the charge density in the laboratory frame is affected by the center-of-mass motion. The center-of-mass eigenproblem of the few-electron system has the same form as the Fock-Darwin equation for the single electron. In the high magnetic field limit, this equation possesses a degenerate ground state (the lowest Landau state), for which the angular momentum is arbitrary. In the case of this degeneracy a superposition of ground states with different angular momenta is still the ground state of the Fock-Darwin equation, even though it leads to solutions with the broken symmetry of the charge density distribution in the laboratory frame. In the HF method, the formation of the Wigner molecule, i.e., the separation of the electrons, is only possible in the broken-symmetry solutions. The results of the present paper show that exact energy is obtained for these broken-symmetry solutions in the infinite magnetic field limit.

In the recent paper of Bednarek et al 37 a study of the accuracy of the HF method has been presented for the quasi-one-dimensional (1D) structure. In the quasi-1D structures the unrestricted HF method becomes exact 37 in the large quantum dots, in which the Wigner molecules are formed 37 . In the 1D structures, the HF method conserves the two-particle parity symmetry of the exact solution; so, there is no problem with the broken symmetry of the HF solutions like in the $2 \mathrm{D}$ circularly symmetric quantum dots.

The present results show that the overestimation of the exact energy obtained in the broken-symmetry MCHF solutions is relatively small at the magnetic fields, for which the ground state is degenerate. The ground state of the few-electron system is twofold degenerate at these magnetic fields, which induce a stepwise change of the ground-state angular momentum (cf. Figs. 4, 6] and 7). In this case, the exact ground state can be a superposition of two states with different angular momenta and therefore the charge density can have the symmetry different from that of the external potential. For these fields the broken symmetry of the HF solutions leads to the decrease of the energy separation between the MCHF and ED results (cf. Fig. 6), which in turn causes the characteristic oscillations of the MCHF error as a function of 
the magnetic field as shown in Figs. 47

The application of the generalized variational basis results in a modification of the phase diagram ${ }^{5}$, for the Wigner molecules. This modification is due to the different precision of the restricted wave function for different phases (cf. Fig. 1). The critical magnetic fields for the MDD breakdown, obtained with the generalized basis, are shifted toward lower values (cf. Fig. 3) and the range of the stability of different phases is modified. The improved results conserve the characteristic features of the original phase diagram ${ }^{5}$ i.e., the intermediate phases correspond to the configurations, for which a larger number of electrons is gathered on the outer ring of the molecule in comparison with the classical, highmagnetic-field, ground-state configuration.

The present calculations performed for small number of electrons indicate that in the high magnetic field the inner charge distribution of electrons can be derived from classical calculations for particles interacting via a Coulomb $(1 / r)$ interaction. On the other hand in the Laughlin wave function ${ }^{38}$, which seems to become an exact description of the many-body state at high magnetic field, the distribution of electrons in the inner coordinates corresponds to classical configuration of particles interacting with a logarithmic potential. It is therefore not excluded that for larger number of electrons the localization may be different than for electrostatically interacting classical particles.

\section{CONCLUSIONS AND SUMMARY}

We have investigated the quantum-dot confined $N$-electron system at high magnetic fields using the HF method with the generalized multicenter basis and the exact diagonalization method. We have indicated that the magnetic-field dependence of the variational parameters of the generalized basis can be used as one of the signatures of the liquid-solid phase transition, i.e., the breakdown of the MDD into the molecular phase. The occurrence of the cusp of the interaction energy as a function of the magnetic field and the decrease of the MCHF angular-momentum expectation value below that corresponding to the MDD are the other signatures of the MDD decay. We have discussed the accuracy of the energy estimates obtained with the broken-symmetry HF solutions, which - in contrast to the exact solutions of the Schrödigner equation - are not eigenfunctions of the total angular-momentum operator. It turns out that the angular-momentum eigenvalues in the MDD phase are reproduced with the high accuracy by the MCHF expectation values, which at higher magnetic fields linearly decrease with increasing $B$ in contrast to the exact stepwise decrease. The results of the present paper show that the MCHF inaccuracy decreases with the increasing magnetic field and that the MCHF method basis yields the exact ground-state energy in the infinite magnetic-field limit. We have found the characteristic oscillations of the HF inaccuracy, which exhibits local minima at those magnetic fields, for which the exact ground state is degenerate with respect to the angular momentum. The relation of these oscillations with the existence of the exact ground states with the broken symmetry has been pointed out. The results of the present paper show that the envelope of the MCHF inaccuracy oscillations at high magnetic field decreases linearly to 0 as a function of $1 / B$ and that in the strictly infinite magnetic field limit the exact energy is obtained for broken-symmetry HF solution with the classical point-charge distribution.

Acknowledgments This work was supported in part by the Polish Government Scientific Research Committee $(\mathrm{KBN})$, the Flemish Science Foundation (FWO-Vl), IUAP, and GOA. One of us (EA) is supported by a EU Marie Curie fellowship. The first author (BS) is supported by the Foundation for Polish Science (FNP).

\section{Appendix}

In order to derive wave function [Eq. (22] let us first consider the single electron in a homogeneous magnetic field. In the Landau gauge, i.e., for $\mathbf{A}(\mathbf{r})=(-B y, 0,0)$, the Hamiltonian has the form

$$
H_{0}=-\frac{\hbar^{2}}{2 m^{*}}\left(\frac{\partial^{2}}{\partial x^{2}}+\frac{\partial^{2}}{\partial y^{2}}\right)+i \hbar \omega_{c} y \frac{\partial}{\partial x}+\frac{m^{*} \omega_{c}^{2}}{2} y^{2},
$$

where $\omega_{c}=e B / m^{*}$. The lowest Landau energy level $E_{0}=\hbar \omega_{c} / 2$ is infinitely-fold degenerate. The corresponding degenerate eigenstates have the form

$$
\chi_{q}(x, y)=C_{1} \exp \left[i q x-(\beta / 2)(y-q / \beta)^{2}\right],
$$

where $\beta=m^{*} \omega_{c} / \hbar=e B / \hbar, C_{1}$ is the normalization constant, and $q \in(-\infty,+\infty)$. Due to this degeneracy, an arbitrary linear combination of wave functions (4) is an eigenfunction of Hamiltonian (33) to eigenvalue $E_{0}$. The most general form of this linear combination can be written as

$$
\psi_{0}(x, y)=\int_{-\infty}^{+\infty} f(q) \chi_{q}(x, y) d q .
$$

Taking

$$
f(q)=\exp \left[q\left(y_{0}-i x_{0}\right)-q^{2} / 2 \beta\right]
$$

and performing the integration in Eq. (5), we obtain

$$
\begin{gathered}
\psi_{0}(x, y)=C_{0} \exp \left\{-(\beta / 4)\left[\left(x-x_{0}\right)^{2}+\left(y-y_{0}\right)^{2}\right]+\right. \\
\left.(i \beta / 2)\left(x-x_{0}\right)\left(y+y_{0}\right)\right\},
\end{gathered}
$$

where $C_{0}$ is the normalization constant. Wave function (77) corresponds to the electron probability density, which is localized around center $\mathbf{r}_{0}=\left(x_{0}, y_{0}\right)$. For arbitrary $\mathbf{r}_{0}$ wave function (7) is the eigenfunction of the Hamiltonian (3) associated with eigenvalue $E_{0}$. Due to the arbitrary 
choice of $\mathbf{r}_{0}$, wave functions (17), localized at different centers, correspond to the same lowest Landau level.

If, in addition to the magnetic field, the electron is subject to the external parabolic potential centered at site $\mathbf{R}=(X, Y)$, i.e.,

$$
V_{\text {conf }}(x, y)=\frac{m^{*} \omega_{0}^{2}}{2}\left[(x-X)^{2}+(y-Y)^{2}\right]
$$

we deal with the Fock-Darwin eigenproblem. Then, the ground-state wave function is centered around $\mathbf{R}$ and is written down in the normalized form as

$$
\begin{gathered}
\psi_{(X, Y) \quad(x, y)}=\exp \left\{-(\alpha / 4)\left[(x-X)^{2}+(y-Y)^{2}\right]+\right. \\
(i \beta / 2)(x-X)(y+Y)\} /(\alpha / 2 \pi)^{1 / 2}
\end{gathered}
$$

where $\alpha=\left(2 m^{*} / \hbar\right) \sqrt{\omega_{0}^{2}+\omega_{c}^{2} / 4}$. Therefore, we obtain the wave function of form (2).
1 A.H. MacDonald, S.R.E. Yang, M.D. Johnson, Aust. J. Phys. 46, 345 (1993)

2 H-M. Müller, S.E. Koonin, Phys. Rev. B 54, 14532 (1996)

3 C. Yannouleas, U. Landman, Phys. Rev. B 61, 15895 (2000)

4 C. Yannouleas, U. Landman, Phys. Rev. B 66, 115315 (2002)

5 B. Szafran, S. Bednarek, and J. Adamowski, Phys. Rev. B 67, 045311 (2003); erratum 67 159902(E) (2003)

${ }^{6}$ B. Szafran, S. Bednarek, J. Adamowski J. Phys. Cond. Matter (2003), 15, 4189 (2003).

7 J. Kainz, S.A. Mikhailov, A. Wensauer, U. Rössler, Phys. Rev. B 65, 115305 (2002)

8 B. Reusch, H. Grabert, Phys. Rev. B 68, 045309 (2003)

9 S.M. Reimann, M. Koskinen, M. Manninen, B.R. Mottelson, Phys. Rev. Lett. 83, 3270 (1999)

10 S.M. Reimann, M. Manninen, Rev. Mod. Phys. 74, 1283 (2002)

11 P.A. Maksym and T. Chakraborty, Phys. Rev. B 45, 1947 (1992).

12 M. Eto, Jpn. J. Appl. Phys. 36, 3924 (1997)

13 H. Immamura, P.A. Maksym, H. Aoki, Physica B 249251, 214 (1998)

14 M. Manninen, S. Viefers, M. Koskinen, S.M. Reimann, Phys. Rev. B 64, 245322 (2001)

15 A. Matulis, F.M. Peeters, Solid State Comm. 117, 655 (2001)

16 S.A. Mikhailov, N.A. Savostianova, Phys. Rev. B 66, 033307 (2002)

17 S.R.E. Yang, A.H. MacDonald, Phys. Rev. B 66, 041304 (2002)

18 A. Wójs, P. Hawrylak, Phys. Rev. B 56, 13227 (1997)

19 M. Dineykhan, R.G. Nazmitdinov, J. Phys. Condens. Matter 11, L83 (1999)
20 B. Szafran, S. Bednarek, J. Adamowski, Phys. Rev. B 67, 115323 (2003)

21 M. Rontani, G. Goldoni, F. Manghi, E. Molinari, Europhys. Lett. 58, 555 (2002)

22 F.M. Peeters, Phys. Rev. B 42, 1486 (1990)

23 T.H. Oosterkamp, J.W. Janssen, L.P. Kouwenhoven, D.G. Austing, T. Honda, S. Tarucha, Phys. Rev. Lett. 82, 2931 (1999)

24 B. Szafran, S. Bednarek, J. Adamowski, Phys. Rev. B 65, 035316 (2002)

25 P.A. Maksym, H. Immamura, G.P. Mallon, H. Aoki, J. Phys. Condens. Matter 12, R299 (2000)

26 R. Egger, W. Häusler, C.H. Mak, H. Grabert, Phys. Rev. Lett 82, 3320 (1999)

27 S. Akbar, I.-H. Lee, Phys. Rev. B 63, 165301 (2001)

28 S. Bednarek, B. Szafran, J. Adamowski, Phys. Rev. B 64, 195303 (2001)

29 K. Maki, X. Zotos, Phys. Rev. B 28, 4349 (1983)

30 R. Price, X. Zhu, S. Das Darma, P.M. Platzmann, Phys. Rev. B 512017 (1999)

31 F. Bolton, U. Rössler, Superlattices Microstruct. 13, 139 (1993)

32 V.M. Bedanov, F.M. Peeters, Phys. Rev. B 49, 2667 (1994)

33 M. Kong, B. Partoens, F.M. Peeters, Phys. Rev. E 65, 046602 (2002)

34 S.M. Girvin, T. Jach, Phys. Rev. B 28, 4506 (1983)

35 P.A. Maksym, Phys. Rev. B 53, 10871 (1996)

36 C.G. Bao, W.Y. Ruan, Y.Y. Liu, Phys. Rev. B 53, 10820 (1996)

37 S. Bednarek, T. Chwiej, J. Adamowski, B. Szafran, Phys. Rev. B 67, 205316 (2003)

38 R.B. Laughlin, Phys. Rev. B 27, 3383 (1983). 